\title{
Translocation of effectors: revisiting the injectosome model
}

Neta Sal-Man', Matthew A Croxen' \& B Brett Finlay ${ }^{\dagger 1}$

'The University of British Columbia, Michael Smith Laboratories, Vancouver, Canada

†Author for correspondence: bfinlay@interchange.ubc.ca

Evaluation of: Akopyan K, Edgren T, Wang-Edgren $\mathrm{H}$ ef al:: Translocation of surface-localized effectors in type III secretion. Proc. Natl Acad. Sci. USA 108(4), 1639-1644 (2011). Type III secretion systems suppress host immune response and modify cell-signaling and regulation pathways by translocation of virulence proteins, called effectors, from the bacteria into the cytosol of the target cells. The common belief was that effectors translocate by a single step mechanism through a continuous channel built up by type III secretion systems. In this article, Akopyan et al. propose an alternative, and possibly parallel, two-step model to translocate effectors into target cells. According to their model, effectors first localized on the surface of the bacterial membrane, followed by a type III secretion system-dependent entry into the host cell.

Many Gram-negative pathogens, including Salmonella, Shigella, Yersinia, enteropathogenic and enterohemorrhagic Escherichia coli and Pseudomonas species, harbor type III secretion systems (T3SSs). These systems are multiprotein export apparatuses that transport effectors from the bacteria into the cytoplasm of the target cell [1]. This translocation process is highly regulated to ensure that secretion occurs at the appropriate time, upon contact with the host cell. The T3SS is described as a nano-syringe, or injectosome, that contains a basal body anchored in the bacterial membrane and an external needle that forms a pore in the plasma membrane of the host cell. Once assembled, the injectosme enables continuous passage of effector proteins from the bacterial cytoplasm across three membranes (two bacterial and one eukaryotic), directly into the host cell. A recent study by Akopyan et al. describes new findings that suggest an alternative translocation model [2]. Immunoelectron microscopy of wild-type Yersinia pseudotuberculosis, in the absence of target cells, detected the effector proteins YopE and YopH primarily on the surface of the bacteria, and not in the cytoplasm, as predicted for many bacterial effectors. This localization is suggested by Akopyan et al. to be the first step in the translocation process. To provide direct evidence that the translocation process can be dissected into two separate steps, purified effectors were extracellularly added and examined for translocation. Using two translocation methods, a $\beta$-lactamase reporter system and a functional assay that detects YopH effects on host cells, they elegantly show that purified YopH that is surface-localized can be translocated into target cells in a T3SS-dependent manner. This translocation process requires the presence of the translocator proteins YopB and YopD. According to their results, they suggest that the translocation process is a two-step process: effector surface-localization (or secretion), followed by translocation.

\section{Discussion}

It has long been recognized that Yersinia effectors are membrane localized. In fact, their name, Yops (Yersinia outer-membrane proteins), originates from where they were first detected - in the outer membrane fraction of bacterial extracts $[3,4]$. An early study by Michiels et al. demonstrated that Yops are truly secreted proteins that reside in the membrane fraction due to their absorption to the bacterial surface [5]. Their ability to associate with the bacterial membrane, as seen in the electron microscopy images of Akopyan et al., was detected only in the absence of target cells, most likely due to their increased extracellular concentration. This unique property of Yops can explain their ability to both be secreted and to coat $Y$. pseudotuberculosis membranes for in vitro studies, as described in Akopyan et al. [2].

The truly exciting result presented in Akopyan et al. is the ability of purified effector to be translocated into host cells in a T3SS-dependent manner. This suggests that surface-localized effectors are still functional and are able to respond to the T3SS when bacteria detect host cells. The ability of a YopH- $\beta$-lactamase fusion protein to translocate to host cells when coated on Salmonella enterica serovar Typhimurium strain further suggests that the two-step model is general and may apply to other pathogens. Nevertheless, there are no other known examples of surface-localized effectors in T3SS-utilizing pathogens. Therefore, additional studies need to be done to determine whether the twostep model is a general mechanism of effector
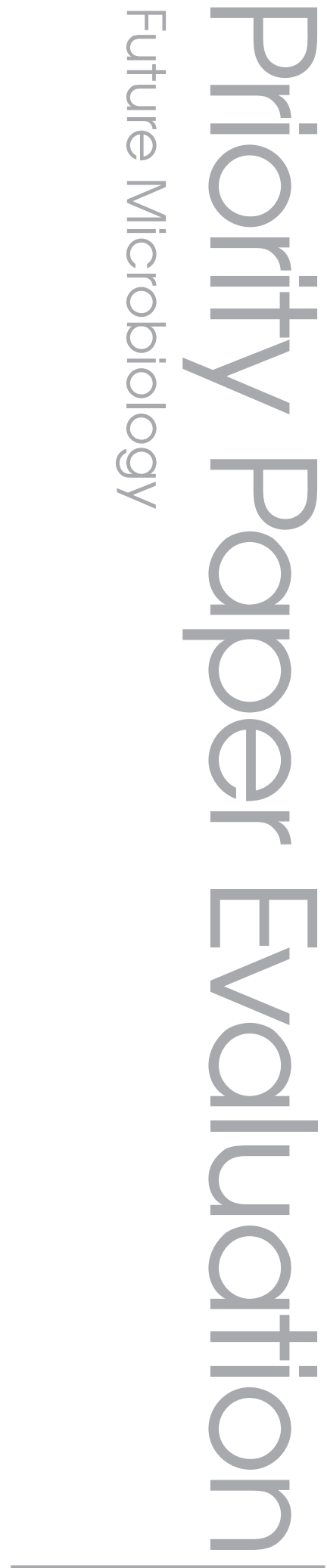

\section{Keywords}

- bacterial pathogenesis

- effectors = type III secretion - Yersinia

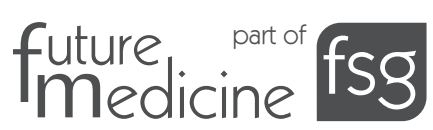


translocation or only true for Yersinia effectors, due to their unique propensity to associate with the membrane fraction.

\section{Conclusion \& future perspective}

Akopyan et al. describe new findings that challenge the accepted mechanism for effector translocation into host cells. In their study, they suggest that effector translocation can proceed through an extracellular intermediate and not necessarily by a one-step model. Based on their findings, it might be possible to use Yersinia coated with effectors as a model system to characterize effectors of less tractable pathogens (e.g., Chlamydia) and their effect on host cells pathways. Although their model is feasible and intriguing, it raises fundamental questions regarding storing effector proteins on the bacterial membrane in terms of energy cost and immune response. Moreover, the precise mechanism for cell-surface localization and whether this localization is T3SS-dependent needs further investigation. Considering this novel model, it will be interesting to determine whether the T3SS forms a continuous channel or has lateral entry sites to enable surface-localized effectors to gain access to the T3SS. Moreover, in light of the new findings of Lara-Tejero et al., which describe a sorting platform in the bacterial cytoplasm to ensure timed secretion of translocators and effectors, it will be important to dissect whether both mechanisms coexist and the significance of using one mechanism over the other [6]. Regardless, we are certain that we are going to witness exciting discoveries in the field of bacterial secretion in the near future.

\section{Financial \& competing interests disclosure}

This work was supported by operating grants to $B B$ Finlay from the Canadian Institutes of Health Research (CIHR) and the Canadian Crohn's and Colitis Foundation. BB Finlay is an HHMI International Research Scholar and the University of British Columbia Peter Wall Distinguished Professor. $N$ Sal-Man is supported by fellowships from Rothschild, the Michael Smith Foundation of Health Research and the Natural Sciences of Engineering Research Council of Canada. MA Croxen is supported by a Canadian Association of Gastroenterology/CIHR/ Ferring Pharmaceuticals fellowship. The authors have no other relevant affiliations or financial involvement with any organization or entity with a financial interest in or financial conflict with the subject matter or materials discussed in the manuscript apart from those disclosed.

No writing assistance was utilized in the production of this manuscript.

\section{Executive summary}

\section{Objective}

- To determine the mechanism of effector translocation in Yersinia pseudotuberculosis.

\section{Methods}

- Electron microscopy to detect effector localization.

- Intracellular $\mathrm{Ca}^{2+}$ measurement in infected neutrophils.

- $\beta$-lactamase translocation reporter assay.

\section{Results}

- YopE and YopH are localized on the surface of Yersinia pseudotuberculosis cells in the absence of host cells.

- Y. pseudotuberculosis coated with purified YopH in vitro block the immediate-early Ca ${ }^{2+}$ response in infected neutrophils.

- Purified YopH- $\beta$-lactamase fusions are translocated into target cells in a T3SS-dependent manner, when coated on $Y$. pseudotuberculosis or Salmonella enterica serovar Typhimurium strains.

- Translocation of YopH- $\beta$-lactamase fusions coated on $Y$. pseudotuberculosis requires a translocation domain that is different from the secretion signal.

\section{Conclusion}

- Y. pseudotuberculosis can translocate the surface-localized effectors YopH and YopK in a T3SS-dependent manner.

\section{Bibliography}

1. Kubori T, Matsushima Y, Nakamura D et al.: Supramolecular structure of the Salmonella typhimurium type III protein secretion system. Science 280(5363), 602-605 (1998).

2. Akopyan $\mathrm{K}$, Edgren T, Wang-Edgren $\mathrm{H}$ et al.: Translocation of surface-localized effectors in type III secretion. Proc. Natl Acad. Sci. USA 108(4), 1639-1644 (2011).
3. Bolin I, Portnoy DA, Wolf-Watz H: Expression of the temperature-inducible outer membrane proteins of Yersiniae. Infect. Immun. 48(1), 234-240 (1985).

4. Portnoy DA, Moseley SL, Falkow S: Characterization of plasmids and plasmidassociated determinants of Yersinia enterocolitica pathogenesis. Infect. Immun. 31(2), 775-782 (1981).
5. Michiels T, Wattiau P, Brasseur R, Ruysschaert JM, Cornelis G: Secretion of Yop proteins by Yersiniae. Infect. Immun. 58(9), 2840-2849 (1990).

6. Lara-Tejero M, Kato J, Wagner S, Liu X, Galan JE: A Sorting platform determines the order of protein secretion in bacterial type III systems. Science 331(6021), 1188-1191 (2011). 